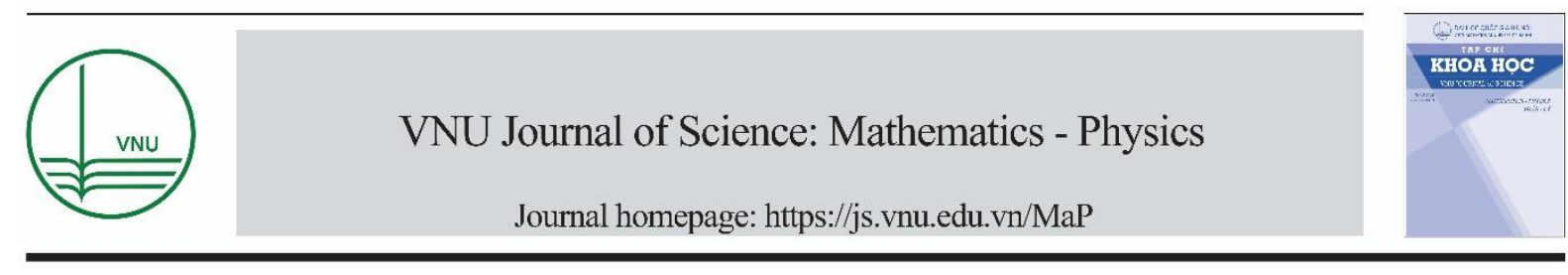

Original Article

\title{
EXAFS Debye-Waller Factors of B2-FeAl Alloys
}

\author{
Nguyen Thi Hong ${ }^{1}$, Nguyen Ba Duc², Ho Khac Hieu, \\ ${ }^{1}$ Hong Duc University, 565 Quang Trung, Dong Ve, Thanh Hoa, Vietnam \\ ${ }^{2}$ Tan Trao University, Km 6, Trung Mon, Yen Son, Tuyen Quang, Vietnam \\ ${ }^{3}$ Duy Tan University, 3 Quang Trung, Hai Chau, Da Nang, Vietnam
}

Received 16 August 2020

Revised 23 September 2020; Accepted 15 October 2020

\begin{abstract}
This work develops the anharmonic correlated Debye model to study the temperaturedependent extended X-ray absorption fine structure (EXAFS) Debye-Waller factors (DWFs) of B2FeAl alloys. We derived the analytical expressions of the EXAFS DWF and Debye frequency as functions of temperature. Numerical calculations were performed for $\mathrm{Fe}_{1-\mathrm{y}} \mathrm{Al}_{\mathrm{y}}$ alloys with various Al concentration ( $\mathrm{y}=0.35,0.40,0.45$ and 0.50$)$ in which $\mathrm{Fe}-\mathrm{Al}$ alloys still maintained $\mathrm{B} 2$ structure. The good agreement between our theoretical results with previous data verifies our developed theory. Our calculations show that DWFs of $\mathrm{Fe}_{1-\mathrm{y}} \mathrm{Al}_{\mathrm{y}}$ alloys increase robustly when temperature and/or $\mathrm{Al}$ concentration in $\mathrm{Fe}_{1-\mathrm{y}} \mathrm{Al}_{\mathrm{y}}$ alloys increase. The increasing of DWF will cause the reduction of the amplitude of EXAFS.
\end{abstract}

Keywords: EXAFS, Debye-Waller factors, Debye model, Anharmonicity, Fe-Al alloys

\section{Introduction}

Extended X-ray absorption fine structure (EXAFS) is an effective method for investigating the structure and thermodynamic properties of crystalline materials [1]. One of the methods to analyze the EXAFS oscillation is the cumulant expansion approach in which second cumulant $\sigma^{2}=\sigma^{(2)}$ is an important factor affecting sensitively amplitudes of EXAFS through the factor $\exp \left(-2 \sigma^{2} k^{2}\right)[2,3]$. The second cumulant, so-called the Debye-Waller factor (DWF), has the form as

\footnotetext{
*Corresponding author.

Email address: hieuhk@duytan.edu.vn
}

https//doi.org/10.25073/2588-1124/vnumap.4597 


$$
\sigma^{2}=\left\langle\left[\vec{R} \cdot\left(\vec{u}_{i}-\vec{u}_{0}\right)\right]^{2}\right\rangle=\left\langle u_{i}^{2}\right\rangle+\left\langle u_{0}^{2}\right\rangle-2\left\langle u_{i} u_{0}\right\rangle,
$$

where, the brackets stand for the thermal average, the unit vector $\vec{R}$ pointing from the Oth site toward the $i$ th site, $\vec{u}_{0}$ and $\vec{u}_{i}$ are the displacements of the $O$ th and the $i$ th sites from their equilibrium positions; $\left\langle u_{i}^{2}\right\rangle$ and $\left\langle u_{0}^{2}\right\rangle$ are the uncorrelated mean-square displacements, while $2\left\langle u_{i} u_{0}\right\rangle$ is the correlation function.

EXAFS and EXAFS cumulants including DWF are sensitive to temperature [4]. This fact can lead to uncertainties in physical information taken from EXAFS. In order to consider the temperaturedependent EXAFS cumulants of crystals, Hung and his collaborators proposed the anharmonic correlated Debye model (ACDM) [5] which has been developed further to study thermal disorder of binary alloys [6]. However, the authors just focused on the specific case of $\mathrm{FeAl}$ alloys, $\mathrm{Fe}_{0.6} \mathrm{Al}_{0.4}$. In this work, we apply the ACDM to calculate and analyze the dependence of EXAFS DWFs of $\mathrm{Fe}_{1-\mathrm{y}} \mathrm{Al}_{\mathrm{y}}$ alloys on temperature and $\mathrm{Al}$ concentration.

\section{Theory}

The ACDM is characterized by an anharmonic effective interaction potential $V_{\text {eff }}(x)$ which is contributed by the oscillation of absorbing and back-scattering atoms, and their neighbors as [5]

$$
V_{\text {eff }}(x)=V(x)+\sum_{j \neq i} V\left(\frac{\mu}{M_{i}} x \hat{\mathbf{R}}_{12} \cdot \hat{\mathbf{R}}_{i j}\right) \cong \frac{1}{2} k_{e f f} x^{2}+k_{3} x^{3}+\cdots,
$$

where $V(x)$ is the interaction potential between absorber and backscatterer; $i=1$ and $i=2$ correspond to absorber and backscatterer, respectively, and the sum $j$ is over all their nearest neighbors, excluding the absorber and backscatterer themselves; $k_{e f f}$ is the effective force constant; $k_{3}$ is the cubic parameter giving an asymmetry in the pair distribution function; $x=r-r_{0}$ is the deviation of instantaneous bond length between the two intermediate atoms from equilibrium.

If we assume the Morse pair potential $V(r)=D\left[e^{-2 \alpha\left(r-r_{0}\right)}-2 e^{-\alpha\left(r-r_{0}\right)}\right]$ can be used to describe the interaction between intermediate atoms, the effective potential for B2 structure can be derived as [6]

$$
V_{\text {eff }}(x) \approx \frac{11}{6} D \alpha^{2} x^{2}-\frac{3}{4} D \alpha^{3} x^{3}+\ldots
$$

where $D$ and $\alpha$ are the Morse potential parameters, $r_{0}$ is the equilibrium distance of two neighbor atoms.

For $\mathrm{Fe}_{1-\mathrm{y}} \mathrm{Al}_{\mathrm{y}}$ alloys, the interatomic effective potential $V_{\text {eff }}$ of system is contributed by both $\mathrm{Fe}-\mathrm{Al}$ pairs and $\mathrm{Fe}-\mathrm{Fe}$ interactions. The increasing of $\mathrm{Al}$ concentration will reduce the number of Fe-Fe nearestneighbor bonds. The anharmonic effective interaction potential $V_{\text {eff }}$ can be approximated as follows: [6]

$$
V_{e f f}=2 y V_{e f f}^{F e A l}+(1-2 y) V_{e f f}^{F e}
$$

where $V_{\text {eff }}^{F e A l}$ and $V_{\text {eff }}^{F e}$ are the effective potential between $\mathrm{Fe}$ and $\mathrm{Al}$ atoms, and between $\mathrm{Fe}$ atoms, respectively. 
From Eqs. (3) and (4), the effective force constant $k_{\text {eff }}$ of $\mathrm{Fe}_{1-\mathrm{y}} \mathrm{Al}_{\mathrm{y}}$ alloys can be derived as

$$
k_{\text {eff }}=2 y k_{\text {eff }}^{F e A l}+(1-2 y) k_{e f f}^{F e},
$$

where

$$
k_{e f f}^{F e}=\frac{11}{3} D_{0}^{F e}\left(\alpha^{F e}\right)^{2} ; \quad k_{e f f}^{F e A l}=\left[1+\frac{5}{3}\left(\mu_{F e}^{2}+\mu_{A l}^{2}\right)\right] D_{0}^{F e A l}\left(\alpha^{F e A l}\right)^{2}
$$

and

$$
\mu_{F e}=\frac{M_{F e}}{M_{F e}+M_{A l}}, \quad \mu_{A l}=\frac{M_{A l}}{M_{F e}+M_{A l}} .
$$

Finally, we obtain the expression of the temperature-dependent EXAFS DWF as $[5,6]$

$$
\sigma^{2}=\left\langle\left(r-r_{0}-\sigma^{(1)}\right)^{2}\right\rangle=\sigma_{0}^{(2)} \int_{0}^{\pi / a} \omega(q) \frac{1+Z(q)}{1-Z(q)} d q,
$$

where $\sigma_{0}^{(2)}=-\frac{\hbar a}{2 \pi} \frac{1}{2 y k_{e f f}^{F e A l}+(1-2 y) k_{e f f}^{F e}} ; q$ is the phonon wave number, $a$ is the lattice constant; $M$ is the mass of composite atoms, the correlated Debye frequency is $\omega_{D}=2 \sqrt{k_{\text {eff }} / M}$; $Z(q)=\exp (\beta \hbar \omega(q))$ with $\omega(q)=2 \omega_{D}\left|\sin \left(\frac{q a}{2}\right)\right|$ is the phonon vibration frequency, $\left|q \leq \frac{\pi}{a}\right|$.

\section{Results and Discussion}

In this section, the expressions derived in the previous section are used to numerically calculate DWFs of $\mathrm{Fe}_{1-\mathrm{y}} \mathrm{Al}_{\mathrm{y}}$ alloys with various $\mathrm{Al}$ concentration $(\mathrm{y}=0.35,0.40,0.45$ and 0.50$)$ in which $\mathrm{Fe}-\mathrm{Al}$ alloys still maintain B2 structure. The Morse potential parameters derived within the Möbius lattice inversion scheme [7], describing the $\mathrm{Fe}-\mathrm{Fe}$ and $\mathrm{Fe}-\mathrm{Al}$ interactions are shown in Table 1.

Table 1. The Morse potential parameters for B2-type $\mathrm{Fe}_{1-\mathrm{y}} \mathrm{Al}_{\mathrm{y}}$ alloys [7].

\begin{tabular}{|c|c|c|c|}
\hline $\mathrm{A}-\mathrm{B}$ & $D_{0}^{A-B}(\AA)$ & $\alpha^{A-B}(\mathrm{eV})$ & $r_{0}^{A-B}\left(\AA^{-1}\right)$ \\
\hline $\mathrm{Fe}-\mathrm{Fe}$ & 0.346 & 2.562 & 2.507 \\
\hline $\mathrm{Fe}-\mathrm{Al}$ & 0.269 & 1.850 & 2.656 \\
\hline
\end{tabular}

Applying our developed ACDM model, we derive the force constants $k_{\text {eff }}, k_{3}$, Debye frequency $\omega_{D}$ (see Table 2), and anharmonic effective potential $V_{\text {eff }}$ of Fe-Al alloys. In Figure 1, we show the anharmonic effective potentials $V_{\text {eff }}$ of $\mathrm{Fe}_{1-\mathrm{y}} \mathrm{Al}_{\mathrm{y}}$ alloys as functions of $x=r-r_{0}$ with various $\mathrm{Al}$ concentration. As can be seen in Figure 1, these potential curves are asymmetric due to the anharmonic contributions described by the third order force constant $k_{3}$ of these potentials. The effective potential curve of FeAl alloys is opened out when $\mathrm{Al}$ concentration increases. These anharmonic effective potentials of FeAl alloys are going to be used for calculating the EXAFS DWF. 


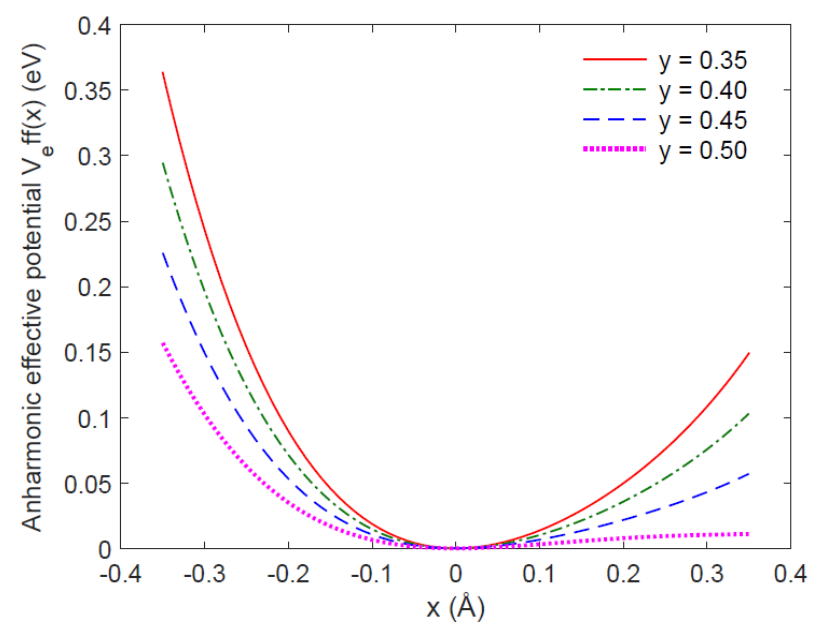

Figure 1. Anharmonic effective potential $V_{\text {eff }}$ of $\mathrm{Fe}_{1-\mathrm{y}} \mathrm{Al}_{\mathrm{y}}$ alloys.

Table 2. The force constants and Debye frequencies of B2-type Fe-Al compounds.

\begin{tabular}{|c|c|c|c|}
\hline Alloys & $k_{\text {eff }}\left(\mathrm{eV} / \AA^{2}\right)$ & $k_{3}\left(\mathrm{eV} / \AA^{3}\right)$ & $\omega_{D}\left({ }^{\prime} 10^{13} \mathrm{~Hz}\right)$ \\
\hline $\mathrm{Fe}_{0.65} \mathrm{Al}_{0.35}$ & 3.1427 & -2.5014 & 5.1528 \\
\hline $\mathrm{Fe}_{0.60} \mathrm{Al}_{0.40}$ & 2.4020 & -2.2353 & 4.5776 \\
\hline $\mathrm{Fe}_{0.55} \mathrm{Al}_{0.45}$ & 1.6613 & -1.9693 & 3.8705 \\
\hline $\mathrm{Fe}_{0.50} \mathrm{Al}_{0.50}$ & 0.9207 & -1.7032 & 2.9311 \\
\hline
\end{tabular}

The temperature dependence of EXAFS second cumulants of $\mathrm{Fe}-\mathrm{Al}$ alloys is presented in Figure 2. It can be observed from this figure that the DWF curves of Fe-Al alloys are almost similar and rise linearly when temperature increases. The rapid development of DWF indicates the significant contributions of thermal lattice vibrations at high temperature. This phenomenon can be explained that when temperature rises the thermal vibration of atoms increases resulting in the enhancement of meansquare relative displacement or DWF, which causes the reduction of the EXAFS amplitude at high temperature. Furthermore, as can be seen from Figure 2, when we increase the Al concentration, the DWFs of $\mathrm{Fe}_{1-\mathrm{y}} \mathrm{Al}_{\mathrm{y}}$ also increases. At temperature lower than $50 \mathrm{~K}$ the values of the second cumulants are very small but different from zero due to the zero-point vibration contributions (a quantum effect). In our calculations, the zero-point contributions to DWFs of $\mathrm{Fe}_{0.65} \mathrm{Al}_{0.35}, \mathrm{Fe}_{0.60} \mathrm{Al}_{0.40}, \mathrm{Fe}_{0.55} \mathrm{Al}_{0.45}$, $\mathrm{Fe}_{0.50} \mathrm{Al}_{0.50}$ alloys are $0.0032 \AA^{2}, 0.0035 \AA^{2}, 0.0040 \AA^{2}$ and $0.0048 \AA^{2}$, respectively.

In Figure 3, due to the lack of experimental EXAFS DWFs of Fe-Al alloys, the change of DWF of FeAl alloy $(\mathrm{y}=0.50)$ is shown. The measurements of the change of EXAFS DWF $\Delta \sigma^{2}$ relative to the lowest temperature value for the first three-shell distances of FeAl have also been presented for comparison [8]. The composition of this bulk sample of FeAl was not analyzed, but the material was weighed before and after melting, with minimal weight loss. The structure was verified by X-ray diffraction with no extraneous peaks. The authors measured EXAFS spectra at beam line X-11 of the National Synchrotron Light Source [8]. As can be observed from Figure 3, our theoretical results of $\mathrm{FeAl}$ alloy $(\mathrm{y}=0.50)$ are consistent with experimental measurements for the second-neighbor shell up to temperature of $300 \mathrm{~K}$. It is worth mentioning that by comparing the change of EXAFS DWF relative 
to the lowest temperature value, we can eliminate errors in calculation of the structural disorder which is caused by strain or alloying.

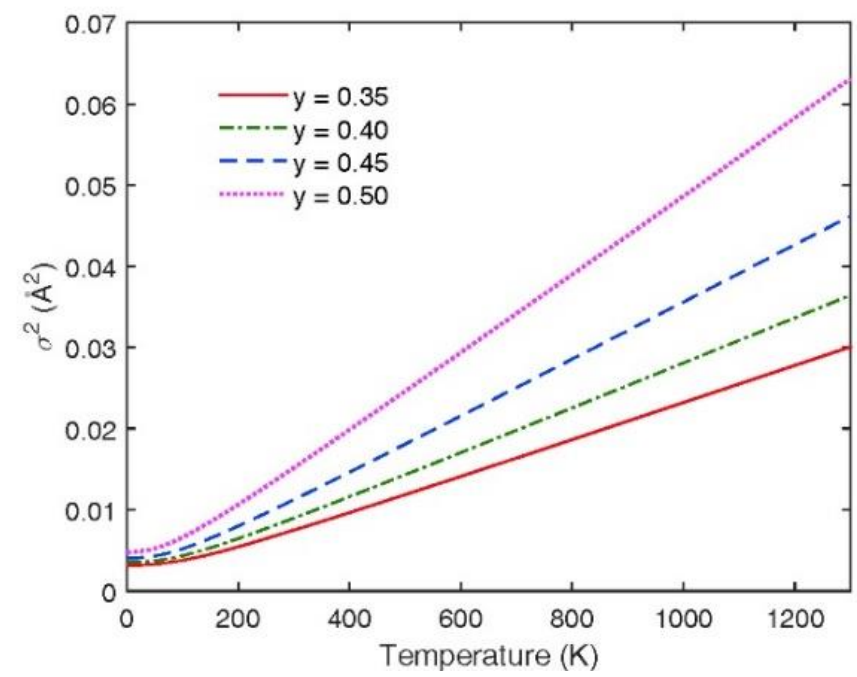

Figure 2. The EXAFS DWFs of $\mathrm{Fe}_{1-\mathrm{y}} \mathrm{Al}_{\mathrm{y}}$ alloys with various $\mathrm{Al}$ concentration.

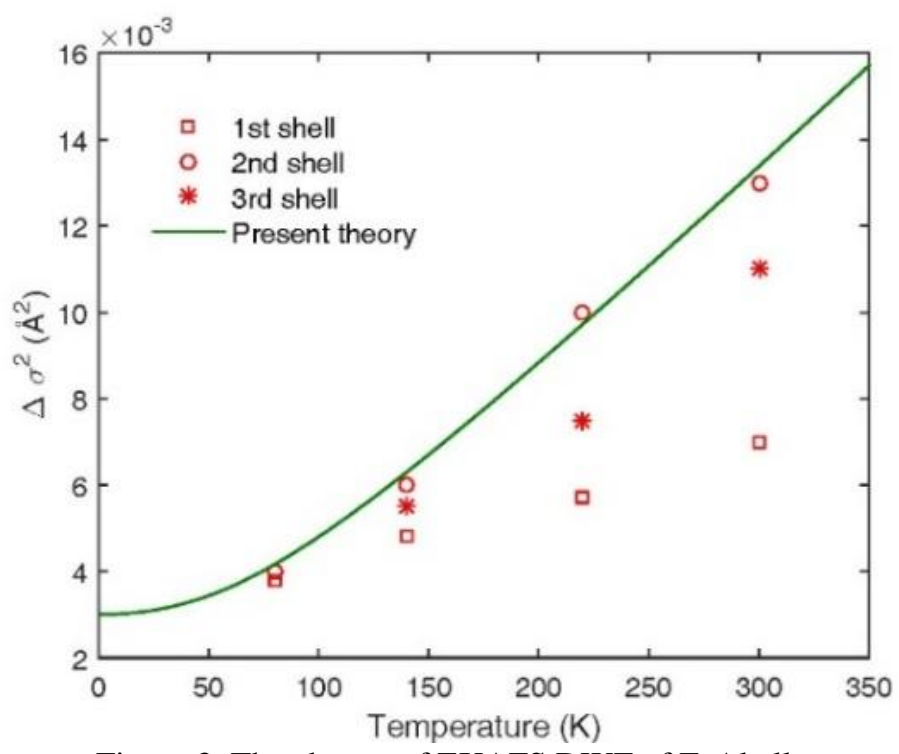

Figure 3. The change of EXAFS DWF of FeAl alloy.

The experimental measurements are shown for comparison [8].

\section{Conclusion}

In this work, we applied the ACDM to investigate the dependence of EXAFS DWFs of $\mathrm{Fe}_{1-\mathrm{y}} \mathrm{Al}_{\mathrm{y}}$ on temperature and $\mathrm{Al}$ concentration. The analytical expressions of the EXAFS DWF and 
Debye frequency have been derived. We performed numerical calculations for $\mathrm{Fe}_{1-\mathrm{y}} \mathrm{Al}_{\mathrm{y}}$ alloys with various $\mathrm{Al}$ concentration $(\mathrm{y}=0.35,0.40,0.45$ and 0.50$)$ in which $\mathrm{Fe}-\mathrm{Al}$ alloys still maintain $\mathrm{B} 2$ structure. The theoretical calculations are in good agreement with those of previous data verifying our developed theory. Our calculations show that the DWFs of $\mathrm{Fe}_{1-\mathrm{y}} \mathrm{Al}_{\mathrm{y}}$ alloys increase rapidly when temperature and/or $\mathrm{Al}$ concentration in $\mathrm{Fe}_{1-\mathrm{y}} \mathrm{Al}_{\mathrm{y}}$ alloys increase. The increasing of DWFs reduces the amplitudes of the EXAFS spectra.

\section{Acknowledgments}

This research is funded by the Vietnam National Foundation for Science and Technology Development (NAFOSTED) under Grant 103.01-2019.55.

\section{References}

[1] Y. Iwasawa, K. Asakura, M. Tada, XAFS Techniques for Catalysts, Nanomaterials, and Surfaces, Springer International Publishing, Switzerland, 2017.

[2] G. Bunker, Application of the Ratio Method of EXAFS Analysis to Disordered Systems, Nucl. Instruments Methods Phys. Res., Vol. 207, No. 3, 1983, pp. 437-444, https://doi.org/10.1016/0167-5087(83)90655-5.

[3] P. Fornasini, F. Monti, A. Sanson, On the Cumulant Analysis of EXAFS in Crystalline Solids, J. Synchrotron Radiat., Vol. 8, No. 6, 2001, pp. 1214-1220, https://doi.org/10.1107/S0909049501014923.

[4] J. J. Rehr, Theoretical Approaches to X-Ray Absorption Fine Structure, Rev. Mod. Phys., Vol. 72, No. 3, 2000 , pp. 621-654, https://doi.org/10.1103/RevModPhys.72.621.

[5] N. V. Hung, N. B. Trung, B. Kirchner, Anharmonic Correlated Debye Model Debye-Waller Factors, Phys. B Condens. Matter, Vol. 405, No. 11, 2010, pp. 2519-2525, https://doi.org/10.1016/j.physb.2010.03.013.

[6] N. T. Hong, H. K. Hieu, N. B. Duc, D. D. Phuong, N. V. Tuyen, D. Q Khoa, Anharmonic Correlated Debye Model for Thermal Disorder in Iron-Rich B2-FeAl Intermetallic Alloy, Vacuum, Vol. 163, 2019, pp. 210-215, https://doi.org/10.1016/j.vacuum.2019.02.023.

[7] C. H. Zhang, S. Huang, J. Shen, N. X. Chen, Structural and Mechanical Properties of Fe-Al Compounds: An Atomistic Study by EAM Simulation, Intermetallics, Vol. 52, 2014, pp. 86-91, https://doi.org/10.1016/j.intermet.2014.04.002.

[8] D. Brewe, D. M. Pease, J. I. Budnick, C. C. Law, Temperature-Dependent Vibrational Properties of NiAl, CoAl, and FeAl $\beta$-Phase Alloys, Phys. Rev. B, Vol. 56, No. 18, 1997, pp. 11449-11455, https://doi.org/10.1103/PhysRevB.56.11449. 